\title{
The identification of the optical/IR counterpart of the 15.8-s transient X-ray pulsar XTE J1946+274
}

\author{
F. Verrecchia ${ }^{1,2}$, G. L. Israel ${ }^{3, \star}$, I. Negueruela ${ }^{4}$, S. Covino ${ }^{5}$, V. F. Polcaro ${ }^{6}$, J. S. Clark ${ }^{7,8}$, I. A. Steele ${ }^{9}$, \\ R. Gualandi ${ }^{10}$, R. Speziali ${ }^{3}$, and L. Stella ${ }^{3, \star}$ \\ 1 Dipartimento di Fisica, Università degli Studi “La Sapienza”, piazza A. Moro 5, 00185 Roma, Italy \\ 2 ASI Science Data Center, ESA-Esrin, via Galileo Galilei, 00044 Frascati, Rome, Italy \\ 3 INAF - Osservatorio Astronomico di Roma, via Frascati 33, 00040 Monteporzio Catone, Italy \\ 4 Observatoire Astronomique de Strasbourg, rue de l'Université 11, 67000 Strasbourg, France \\ 5 INAF - Osservatorio Astronomico di Brera, via E. Bianchi 46, 23807 Merate, Italy \\ 6 Istituto di Astrofisica Spaziale, CNR, Area della Ricerca di Roma Tor Vergata, via Fosso del Cavaliere, 00133 Roma, Italy \\ 7 Astronomy Centre, CPES, University of Sussex, Brighton BN1 9QH, UK \\ ${ }^{8}$ Dept. of Physics \& Astronomy, University College London, London WC1E 6BT, UK \\ 9 Astrophysics Research Institute, Liverpool John Moores University, Liverpool CH41 1LD, UK \\ ${ }^{10}$ INAF - Osservatorio Astronomico di Bologna, via Ranzani, 1, 40127, Bologna, Italy
}

Received 15 May 2002 / Accepted 24 July 2002

\begin{abstract}
We report on the discovery of the optical/IR counterpart of the $15.8 \mathrm{~s}$ transient X-ray pulsar XTE J1946+274. We re-analyzed archival BeppoSAX observations of XTE J1946+274 obtaining a new refined position (a circle with 22" radius at $90 \%$ confidence level). Based on this new position we carried out optical and infra-red (IR) follow-up observations. Within the new error circle we found a relatively optical faint $(B=18.6)$ IR bright $(H=12.1)$ early type reddened $\operatorname{star}(V-R=1.6)$. The optical spectra show strong $\mathrm{H} \alpha$ and $\mathrm{H} \beta$ emission lines. The IR photometric observations of the field confirm the presence of an IR excess for the $\mathrm{H} \alpha$-emitting star $(K=11.6, J-H=0.6)$ which is likely surrounded by a circumstellar envelope. Spectroscopic and photometric data indicate a B0-1V-IVe spectral-type star located at a distance of 8-10 kpc and confirm the Be-star/X-ray binary nature of XTE J1946+274.
\end{abstract}

Key words. stars: individual: - XTE J1946+274; GRO J1944+26 - binaries: general - stars: pulsars: general stars: emission-line, $\mathrm{Be}-\mathrm{X}$-rays: stars

\section{Introduction}

High Mass X-ray Binary systems (HMXRBs) hosting a neutron star (NS) are a class of intrinsically variable and bright $\mathrm{X}$-ray sources whose luminosity is powered by accretion from a massive companion, often a $B$-emission spectral-type star (BeXRBs; White et al. 1995; Coe 2000).

BeXRBs usually show in the X-ray emission three typical behaviours (sometimes more than one within the same source): (i) bright $\mathrm{X}$-ray transients characterized by giant outburst with luminosity up to $L_{x}=10^{38} \mathrm{erg} \mathrm{s}^{-1}$ (Type II; Stella et al. 1986), unrelated to the orbital phase and with high spin-up rates, (ii) transients characterized by periodic outbursts of relatively high luminosity ( $L_{x} \simeq 10^{36}-10^{37} \mathrm{erg} \mathrm{s}^{-1}$; Type I) generally occurring at periastron passage of the NS, and (iii) sources which do not display outbursts, but are moderately variable (of a factor

\footnotetext{
Send offprint requests to: F. Verrecchia, e-mail: verrecchia@mporzio.astro.it

* Affiliated to I.C.R.A.
}

up to 10-100) and show a low luminosity $\left(L_{x} \leq 10^{36} \mathrm{erg} \mathrm{s}^{-1}\right)$ pulsed persistent emission (Negueruela 1998).

Be stars are early type stars, with mass in the range of 2-20 $M_{\odot}$ and luminosity class III to $\mathrm{V}$, which often display Balmer lines in emission in their optical spectra. These stars are characterized by high rotational velocity (up to $\sim 70 \%$ of their break-up velocity), and by phenomena of equatorial mass ejection at irregular times that give rise to a rotational-dominated quasi-Keplerian decretion disc (Hanuschik 1996; Porter 1999; Okazaki 2001; Okazaki \& Negueruela 2001). The widely accepted empirical model interprets the $\mathrm{X}$-ray emission from these systems, in the three mentioned behaviours, as due to sporadic mass accretion of the NS during the expansion phase of the Be disc, or to periodic close encounters of the NS in highly eccentric orbit with the dense circumstellar envelope around the Be star.

At optical wavelengths these stars are difficult to classify owing to the presence of emissions lines produced by the circumstellar envelope and to the high absorption column that affect systems lying in the Galactic plane. So far only about 
30 optical counterparts of BeXRB's have been discovered out of the $>100$ known and $10^{4}-10^{5}$ expected (Nelson et al. 1993, 1995; Covino et al. 2000; Covino et al. 2001; Israel et al. 2000a; Israel et al. 2000b; Israel et al. 2001; Chakrabarty et al. 2002).

The hard X-ray transient source XTEJ1946+274 was discovered with the All Sky Monitor (ASM) on board the Rossi XTE satellite during a scan of the Vul-Cyg region on 1998 September 5 (Smith \& Takeshima 1998). XTE J1946+274 lies inside the error box of the Ariel V transient 3A 1942+274 discovered in the 1976 (Warwick et al. 1981). Campana et al. (1999) have estimated a chance probability of $\sim 7 \%$ that XTE J1946+274 and 3A 1942+274 are not the same source (assuming $\sim 1000$ hard X-ray transients in the Galaxy). The observed flux raised from $\sim 13$ mCrab (2$12 \mathrm{keV}$ ) on September 5 to $\sim 60 \mathrm{mCrab}$ on September 15 , to $\sim 110 \mathrm{mCrab}$ on September 16 (in 2-60 keV; Smith \& Takeshima 1998). Further observations carried out by BATSE (Wilson et al. 1998) led to the detection of X-ray pulsations with a period of $15.83 \pm 0.02 \mathrm{~s}$, later confirmed through pointed Rossi XTE/PCA observations (Smith \& Takeshima 1998). A study of the lightcurve of RXTE/ASM has revealed an $\sim 80 \mathrm{~d}$ (or $160 \mathrm{~d}$ ) X-ray modulation, interpreted as orbital modulation (Campana et al. 1999).

The 1998 outburst was also monitored through a BeppoSAX campaign (Campana et al. 1998) which revealed a complex pulse profile (in $1-10 \mathrm{keV}$ ) and a complex continuum spectrum, with an iron line at $6.6 \mathrm{keV}$ and an inferred column density of $\sim 1.6 \times 10^{22} \mathrm{~cm}^{-2}$. Using Rossi XTE data, a cyclotron line has been detected at $\sim 35 \mathrm{keV}$ with a FWHM of $\sim 8 \mathrm{keV}$ (Heindl et al. 2001), implying a magnetic field strenght of about $3.1(1+z) \times 10^{12} \mathrm{G}$, where $z$ is the gravitational redshift of the scattering region.

In this paper we report on the identification of the optical/IR counterpart of XTEJ1946+274 with a $R \sim 15$, Be spectraltype star. Based on BeppoSAX observations (circular uncertainty region with a radius of $40^{\prime \prime}$ ), we detected a reddened ( $V-R=1.6)$ star, showing in the optical spectrum a strong $\mathrm{H} \alpha$ emission line. The field of XTEJ1946+274 has been subsequently observed in the IR band. The candidate counterpart resulted as the second brightest object in the BeppoSAX error circle $(H \sim 12 \mathrm{mag})$, while the detection of Bracket $\mathrm{H} \gamma$ emission lines in the IR spectra confirmed the Be spectral classification of the star. A recently refined BeppoSAX error circle (22" of radius) has finally confirmed the identification ruling out the possible link between the $\mathrm{X}$-ray transient pulsar and the brightest IR source included in the previous BeppoSAX uncertainty region.

\section{The $\mathrm{X}$-ray position}

The positional uncertainty region obtained with the Rossi XTE/PCA is a circle with a radius of $2^{\prime} .4(90 \%$ confidence level; Takeshima \& Chakrabarty 1998); the latter was reduced with BeppoSAX Narrow Field Instruments pointed observations to 40" (90\% confidence level; Campana et al. 1998). The BeppoSAX X-ray position was RA = $19^{\mathrm{h}} 45^{\mathrm{m}} 38^{\mathrm{s}}$, Dec $=+27^{\circ} 21$ '. (equinox 2000.0). This position could be refined thanks to a detailed study performed on

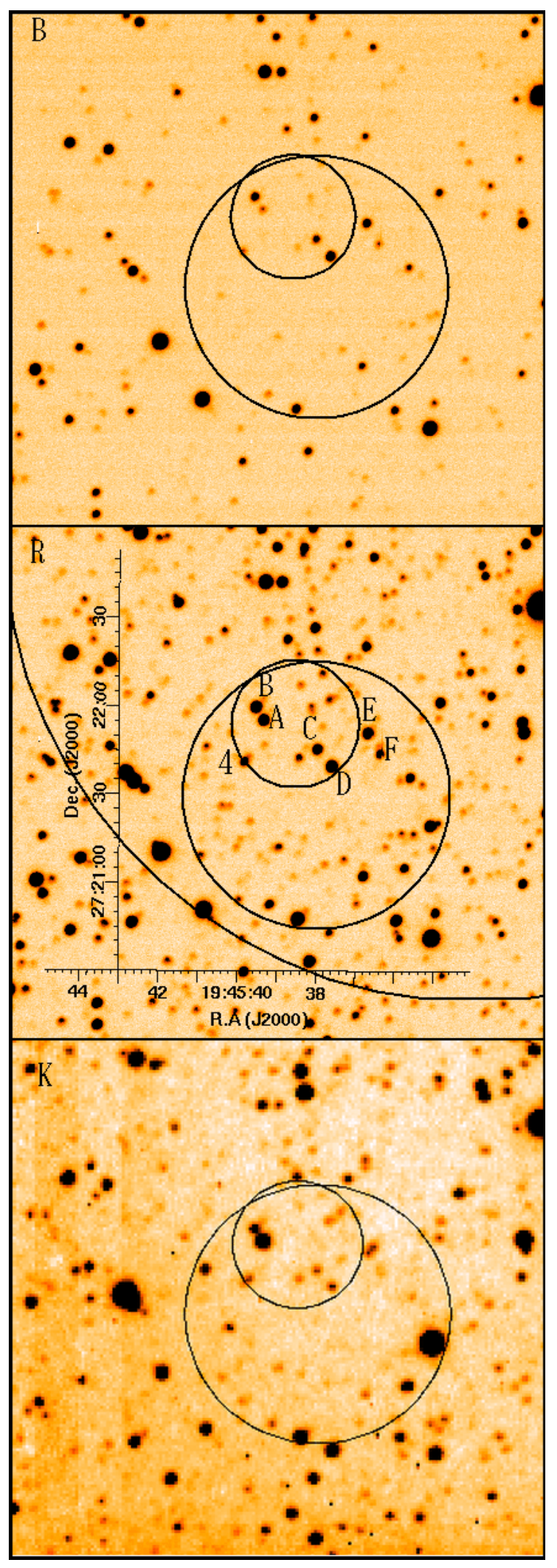

Fig. 1. Optical $B, R$-filter (upper panels) and IR $K$-filter (lower panel) images of the field of XTE J1946+274 together with the X-ray position uncertainty circles derived from BeppoSAX (inner circles) and Rossi XTE (larger circle in the $R$-filter image) observations; RA (hms) and Dec $\left({ }^{\circ}, \prime \prime\right)$ coordinates are shown in the central panel (equinox J2000). The object marked with A is the proposed optical counterpart. 
Table 1. Optical/IR observations carried out for the field of XTE J1946+274.

\begin{tabular}{|c|c|c|c|c|c|c|}
\hline Telescope \& Instrument & Date & $\begin{array}{l}\text { Exp. } \\
\text { (s) }\end{array}$ & $\begin{array}{l}\text { Seeing } \\
\left({ }^{\prime \prime}\right)\end{array}$ & $\begin{array}{c}\text { Range/Slit } \\
\left(\AA /{ }^{\prime \prime}\right)\end{array}$ & Candidate & $\begin{array}{l}\text { Cand. A H } \alpha E W \\
(\AA)\end{array}$ \\
\hline $1.5 \mathrm{~m}$ Cassini \& BFOSC & 1999 July 11 & 2400 & 2.5 & $4000-9000 / 2.5$ & $\overline{C, D}$ & - \\
\hline- & 1999 July 12 & 150 & 1.9 & $V$ & & - \\
\hline- & $”$ & 300 & 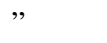 & $B$ & & - \\
\hline - & ” & 100 & $”$ & $R$ & & - \\
\hline - & $"$ & 60 & $"$ & $I$ & & - \\
\hline- & $"$ & 1800 & $"$ & $4000-9000 / 2.5$ & $\mathrm{~B}, \mathrm{~A}$ & $-39 \pm 7$ \\
\hline- & 1999 July 13 & 2400 & 3.3 & $4000-9000 / 2.5$ & E,F & \\
\hline - & $"$ & 1800 & 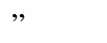 & $"$ & $\mathrm{~B}, \mathrm{~A}$ & $-38 \pm 7$ \\
\hline- & 1999 July 14 & 2400 & 2.0 & $4000-9000 / 2.0$ & 4 & - \\
\hline- & $"$ & 5400 & $"$ & ", & A & $-40 \pm 10$ \\
\hline- & 1999 July 15 & 2400 & 2.6 & $4000-9000 / 2.5$ & A & $-40 \pm 12$ \\
\hline - & 1999 July 16 & 4500 & 2.0 & $3300-5800 / 2.5$ & A & - \\
\hline- & 1999 July 17 & 2700 & 1.6 & " & A & - \\
\hline- & 1999 July 19 & 2700 & 1.5 & $5800-8300 / 2.5$ & A & $-42 \pm 3$ \\
\hline- & " & 2700 & $"$ & $5800-8300 / 2.0$ & A & $-40 \pm 4$ \\
\hline- & " & 1800 & $"$ & $4000-9000 / 2.5$ & A & $-45 \pm 8$ \\
\hline $3.8 \mathrm{~m}$ UKIRT \& CGS4 & 1999 July 24 & 300 & 1.5 & $20400-22000 / 1.0$ & A & - \\
\hline 1.1 m AZT-24 \& SWIRCAM & 1999 July 27 & 60 & 2.1 & $J$ & & - \\
\hline- & 1999 July 27 & 60 & $"$ & $H$ & & - \\
\hline- & 1999 July 27 & 60 & ” & $K$ & & - \\
\hline $4.2 \mathrm{~m}$ WHT \& ISIS & 2000 July 18 & 2400 & 0.9 & $3200-9000 / 1.0$ & A & $-41.0 \pm 0.5$ \\
\hline $1.5 \mathrm{~m}$ Cassini \& BFOSC & 2000 July 31 & 3600 & 2.0 & $5000-9500 / 2.5$ & A & $-40 \pm 8$ \\
\hline- & 2000 August 28 & 1800 & 3.0 & $4000-9000 / 2.5$ & A & $-33 \pm 5$ \\
\hline- & 2001 June 18 & 3600 & 1.8 & $3600-8000 / 2.0$ & A & $-40 \pm 5$ \\
\hline
\end{tabular}

73 relatively bright sources with known optical position observed by BeppoSAX during its life (see also Fiore et al. 2001). The position uncertainty was found to depend on several factors (intensity, off-axis angle, roll angle, energy-dependent PSF, number of gyros and star-treakers, etc.) some of which can be taken properly into account ending up with a MECS error radius of $14^{\prime \prime}$ and $22^{\prime \prime}$ at $68 \%$ and $90 \%$ confidence level, respectively. The first BeppoSAX position was than corrected for these systematic uncertainties by performing a simple boresight correction: the final XTEJ1946+274 position is $\mathrm{RA}=19^{\mathrm{h}} 45^{\mathrm{m}} 38^{\mathrm{s}} .5, \mathrm{Dec}=+27^{\circ} 21^{\prime} 54^{\prime \prime} .3$ (radius of $22^{\prime \prime}$ at $90 \%$ confidence level, equinox 2000.0).

\section{Optical/IR observations}

We carried out observations in the optical and $I R$ band of the BeppoSAX error circle in several observing periods during 1999-2001 (see Table 1) at the Loiano observing station (Bologna Observatory), at Campo Imperatore (Observatory of Rome), at the United Kingdom Infrared Telescope (UKIRT, Mauna Kea, Hawaii) and at the William Herschel Telescope (WHT, La Palma, Spain).

\subsection{Imaging}

On July 1999 we performed imaging and photometry in the $B(300 \mathrm{~s}), V(150 \mathrm{~s}), R(100 \mathrm{~s})$ and $I(60 \mathrm{~s})$ filters with the $1.5-\mathrm{m}$ Cassini telescope equipped with the Bologna Faint Objects Spectrometer and Camera (BFOSC; Bregoli et al. 1987; Merighi et al. 1994; 12!2 × 12!2 field of view and
$0.58 /$ pixel resolution, and a maximum resolution of $\sim 900$ in the 3500-9000 Å range with a 1'.'0 slit). Data reduction was performed using standard ESO-MIDAS procedures for bias subtraction and flat-field correction. Aperture and profile-fitting photometry for each stellar object in the image was derived with the DAOPHOT II program (Stetson 1987).

We performed astrometry of the field using the USNO cata$\log$, resulting in a better than 0.44 positional accuracy. The same field was observed in the IR $J(60 \mathrm{~s}), H(60 \mathrm{~s})$ and $K(60 \mathrm{~s})$ filters on July 1999 with the 1.1-m AZT-24 telescope equipped with the Supernova Watchdogging IR Camera (SWIRCAM; $4.4 \times 4.4$ field of view and 1'.04/pixel resolution). Similar analysis procedures were applied to IR data.

Figure 1 shows the $B, R$ and $K$-filter images of the field which includes the XTE J1946+274 position: the $90 \%$ position uncertainty circles as inferred by Rossi XTE (the largest one in the $R$-filter image) and BeppoSAX (the inner circles) are also shown.

Within the refined BeppoSAX X-ray positional error circle we detected seven relatively bright (see Fig. 1) stars among which only one (labeled as A) was a reddened $(V-R=1.6$, all others had $V-R<1)$ and relatively bright IR object $(H=$ 12.1). In Table 2 we list results of optical/IR photometry for all seven stars.

\subsection{Optical spectroscopy}

During July 1999 we also performed low-resolution $(\sim 18 \AA)$ spectroscopy of all relatively bright stars within the refined BeppoSAX circle (see Table 1). We applied standard 
Table 2. Optical and IR results for the objects in the BeppoSAX error circle. Object A is the proposed counterpart.

\begin{tabular}{cccccccccc}
\hline \hline Object & $\begin{array}{c}B \\
( \pm 0.05)\end{array}$ & $V$ & $\begin{array}{c}R \\
( \pm 0.03)\end{array}$ & $\begin{array}{c}I \\
( \pm 0.02)\end{array}$ & $V-R$ & $\begin{array}{c}J \\
( \pm 0.1)\end{array}$ & $\begin{array}{c}H \\
( \pm 0.1)\end{array}$ & $\begin{array}{c}K \\
( \pm 0.1)\end{array}$ & $J-H$ \\
\hline $\mathrm{A}$ & 18.62 & $16.90 \pm 0.03$ & 15.32 & 13.32 & 1.58 & 12.7 & 12.1 & 11.6 & 0.6 \\
$\mathrm{~B}$ & 17.29 & $15.53 \pm 0.03$ & 15.13 & 13.74 & 0.94 & 13.9 & 13.3 & 13.2 & 0.6 \\
$\mathrm{C}$ & 17.71 & $16.54 \pm 0.04$ & 15.68 & 14.37 & 0.86 & 14.7 & 14.2 & 14.1 & 0.5 \\
$\mathrm{D}$ & 16.71 & $15.71 \pm 0.03$ & 14.96 & 13.76 & 0.75 & 14.3 & 13.9 & 14.0 & 0.4 \\
$\mathrm{E}$ & 17.15 & $16.06 \pm 0.03$ & 15.33 & 14.08 & 0.73 & 14.5 & 13.9 & 13.8 & 0.6 \\
$\mathrm{~F}$ & 18.89 & $17.33 \pm 0.04$ & 16.50 & 15.16 & 0.83 & 15.7 & 15.2 & 15.1 & 0.5 \\
4 & 18.94 & $17.56 \pm 0.03$ & 16.66 & 15.22 & 0.90 & - & - & - & - \\
\hline RA(J2000) $=$ & $19^{\mathrm{h}} 45^{\mathrm{m}} 39.3$ & & & & & & & \\
Dec(J2000) $=$ & $+27^{\circ} 21^{\prime} 55^{\prime \prime} 4$ & & & & & & & \\
Spectral type: & B0-B1 IV-V & & & & & & \\
$E_{B-V}:$ & $1.9 \rightarrow 2.2$ & & & & & & & \\
Distance: & $\sim 8-10 \mathrm{kpc}$ & & & & & &
\end{tabular}

Note - Photometric uncertainties are at 3-sigma confidence level. Position uncertainty is $0{ }^{\prime} 4$.

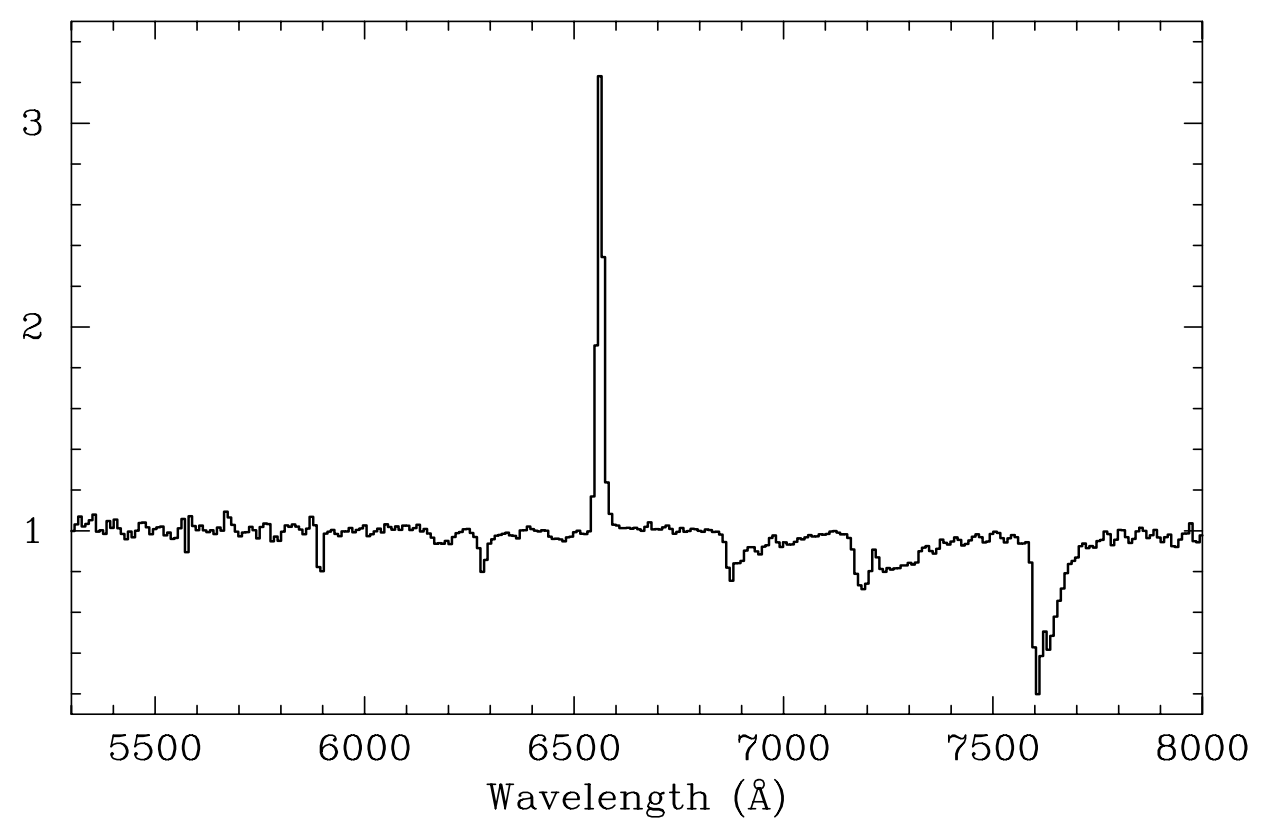

Fig. 2. The 3600-s low resolution (18 ̊) spectrum of XTE J1946+274 candidate A (see Fig. 1 middle panel) obtained on 2000 July 31 from the $1.5 \mathrm{~m}$ Cassini telescope at Loiano (Bologna, Italy).

corrections to spectra, i.e. one-dimensional stellar and sky spectra extraction, then removed cosmic rays and, when weather condition were satisfactory, we performed absolute calibration both for spectroscopy and photometry.

All the spectra carried out in July 1999 in the 4000-9000 band (with different slit apertures) were rebinned to the largest resolution, $18.4 \AA$, and then summed to increase the $\mathrm{S} / \mathrm{N}$ ratio. None of the objects showed emission lines except star A. For the latter source we detected a strong emission line (equivalent width, $E W$, of $-43 \pm 5 \AA$ and Full Width Half Maximum, FWHM, comparable with the spectral resolution) with central wavelength corresponding to that of $\mathrm{H} \alpha$ (see e.g. Fig. 2 for a 5000-9500 ^ spectrum of star A obtained during a 3600 s observation performed on 2000 July 31 ). Similarly we reduced, rebinned ( $9 \AA$ of resolution) and summed two spectra of star A in the band 5800-8300 $\AA$ to better characterize the $\mathrm{H} \alpha$ emission line $(E W-42 \pm 2 \AA)$.
Spectra for star A were subsequently carried out routinely during several 2000-2001 observational nights to study the possible presence of variations of emission line parameters. The $\mathrm{H} \alpha E W \mathrm{~s}$ are consistent with being constant during our observations (see Table 1). Owing to the faintness of the source shortwards of $5000 \AA$, no emission lines have been detected in the blue part of spectra, with the exception of a possible emission $(E W-6.0 \pm 0.3 \AA)$ corresponding to $\mathrm{H} \beta$.

Finally, on 2000 July 18 two 1200-s medium-resolution spectra were taken with the Intermediate Dispersion Spectroscopic and Imaging System (ISIS) mounted on the $4.2 \mathrm{~m}$ William Herschel Telescope (WHT), located at the Observatorio del Roque de los Muchachos, (La Palma, Spain). The blue arm was equipped with the R300B grating and the EEV\#10 CCD, which gives a nominal dispersion of $\sim 0.9 \AA /$ pixel. The resolution at $\mathrm{H} \alpha$, estimated from the FWHM of arc lines, is $\sim 3.3 \AA$. The red arm was equipped 


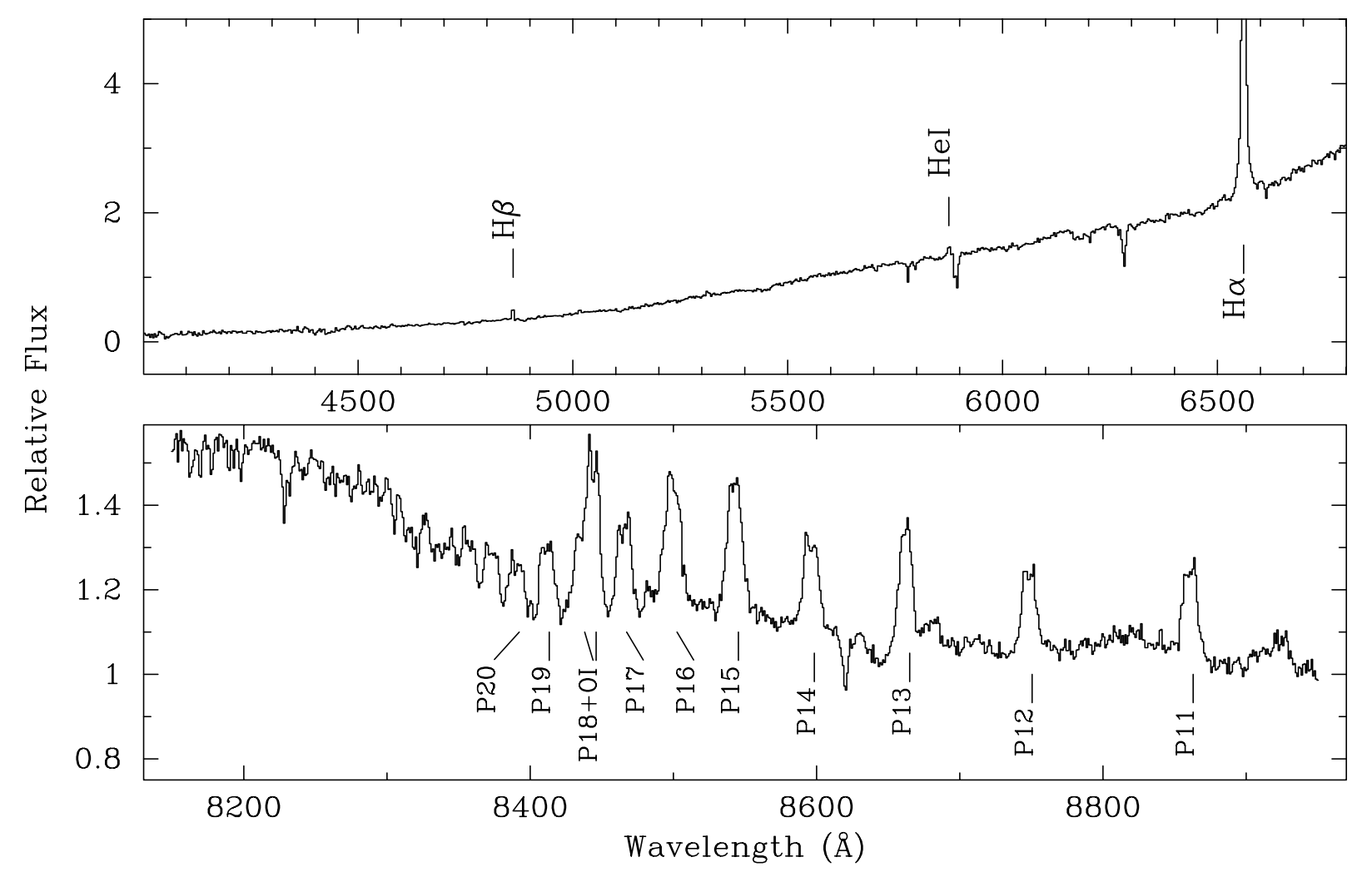

Fig. 3. The 2400-s spectrum ( $3.3 \AA$ and $1.6 \AA$ spectral resolution in the 4000-7000 $\AA$ and $8000-9000 \AA$ range, respectively) of XTEJ1946+274 candidate A obtained on 2000 July 18 from the $4.2 \mathrm{~m}$ William Herschel Telescope at Roque de los Muchachos (La Palma, Spain). Balmer, Paschen and helium series lines are indicated.

with the R600R grating and the Tek4 CCD, which gives a nominal dispersion of $\sim 0.8 \AA /$ pixel (the resolution is $\sim 1.6 \AA$ at $\lambda \sim 8500 \AA$ ). The blue part of the spectrum is dominated by strong diffuse absorption bands (see Fig. 3). The only feature clearly recognizable shortwards of $\mathrm{H} \beta$ (which is clearly in emission with $E W$ of $-2.0 \pm 0.5 \AA$ ) is the $\lambda 4430 \AA$ DIB. An exact spectral classification is therefore impossible. However, based on the usual properties of Be stars, we can strongly constrain the spectral type. The presence of He I $\lambda 5875 \AA$ strongly in emission is only observed in the earliest Be stars (earlier than B2). Likewise, the presence of strong emission in the Paschen series (labelled with P in the lower panel of Fig. 3) confirms that the object is earlier than B2 (Andrillat et al. 1988). As the lack of a detectable He II $\lambda 5412 \AA$ absorption line imposes a spectral type later than 09.5 , the object is constrained to lie in the B0-B1 range. This is within the spectral range occupied by known Galactic and LMC Be/X-ray binary counterparts, which have spectral types tightly concentrated around B0 (Negueruela \& Coe 2002).

\subsection{IR spectroscopy}

Spectroscopic IR observations of XTE J1946+274 candidate were made with the United Kingdom Infrared Telescope (UKIRT; Joint Astronomy Centre, Mauna Kea, Hawaii) on 1999 July 24, using the Cooled Grating Spectrometer (CGS4). Observations between 2.04-2.2 microns were made using the long focal length camera plus the 150 line/mm grating,

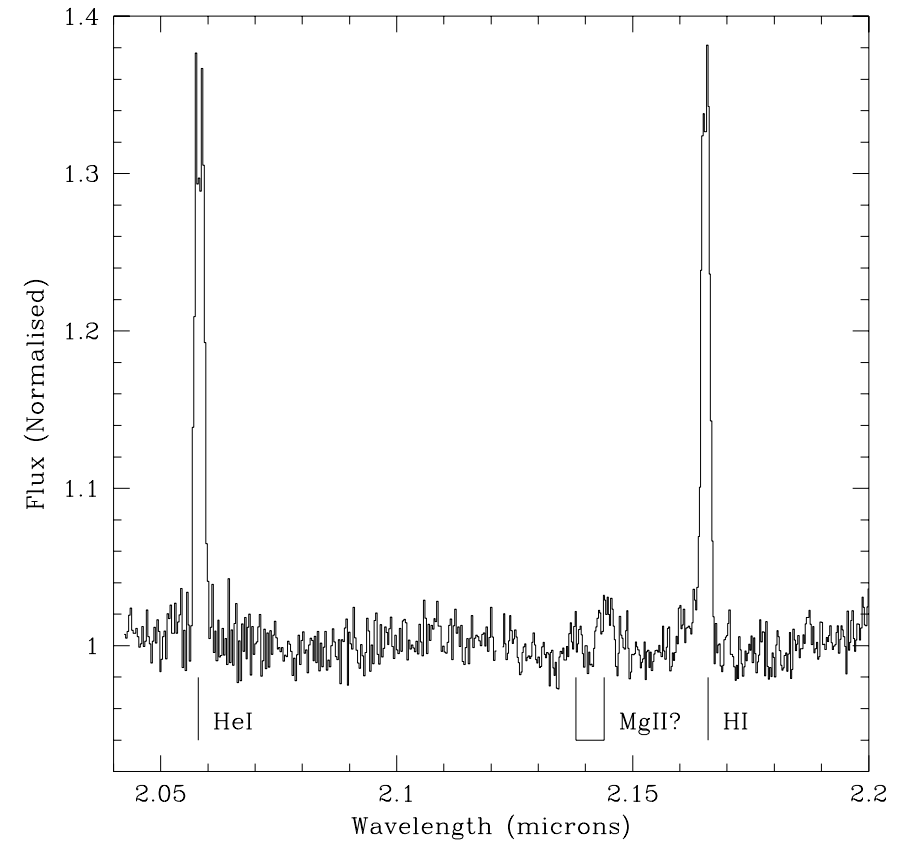

Fig. 4. The 300-s IR spectrum of XTE J1946+274 candidate A obtained on 1999 July 24 from the UKIRT (Maunea Kea, Hawaii).

giving a velocity resolution of $\sim 50 \mathrm{~km} \mathrm{~s}^{-1}$. Initial data reduction was carried out at the telescope using the CGS4DR software (Puxley et al. 1992). This removes bad pixels, debiases, flat-fields, linearity corrects and interleaves oversampled scan 
positions. The subsequent stages of data reduction, comprising of sky subtraction, extraction, derippling and wavelength calibration, were carried out using the Starlink-supported package FIGARO. Removal of telluric features was accomplished via the procedure described by Clark \& Steele (2000).

The spectrum (see Fig. 4) shows strong emission from $\mathrm{He}_{\mathrm{I}}$ $2.058 \mu \mathrm{m}(E W=-8.1 \pm 0.4 \AA)$ and H I Bracket $\gamma(E W=$ $-8.0 \pm 0.4 \AA$ ). FWHMs for the two lines are identical within the errors on the measurements and yield a mean value of $300 \pm$ $50 \mathrm{~km} \mathrm{~s}^{-1}$. There is some evidence for weak emission in the $\mathrm{Mg}_{\text {II }} 2.138 / 2.144 \mu \mathrm{m}$ doublet $(E W=-0.6 \pm 0.4 \AA$ and $-1.4 \pm$ $0.4 \AA$, respectively), although further higher $\mathrm{S} / \mathrm{N}$ observations are required to confirm this. If the emission features are real the ratio of the $E W$ of both lines suggests that the lines are optically thin, and are expected to be excited via $\operatorname{Ly} \beta$ fluorescence. No evidence for emission from Fe II $2.189 \mu$ m or He $\mathrm{I}_{2} .112 / 3 \mu \mathrm{m}$ emission or absorption was found.

Comparison to the $K$ band spectra of classical Be stars presented by Clark \& Steele (2000) suggests that XTE J1946+274 candidate A can be classified as a Group 1 object consistent with it being of early (O9-B3) spectral type (given the paucity of photospheric features in the near IR it is not possible to classify a Be star to greater accuracy than \pm 2 spectral types or provide a luminosity class) in agreement with the optical spectroscopic findings. We find no anomalous emission from higher excitation species to suggest that the star has a compact companion; in line with the trend displayed by other Be/X-ray binaries for which $K$ band spectra have been obtained which also have spectra indistinguishable from those of isolated early spectral type classical Be stars (e.g. Clark et al. 1999).

\section{Discussion and conclusion}

We have presented the X-ray, optical and IR observational data of the field containing the error circle of XTE J1946+274; these observations led to the likely identification of the optical counterpart of this 15.8-s transient X-ray pulsar. Optical/IR photometric measurements are consistent with a relatively reddened and distant blue star, similar to known optical/IR counterparts of BeXRBs (see Nelson et al. 1993, 1995; Israel et al. 2000a; Israel et al. 2000b; Covino et al. 2000; Covino et al. 2001; Israel et al. 2001; Chakrabarty et al. 2002).

Owing to the uncertainty in the spectral classification, the measurement of the distance from optical data becomes difficult; it is however possible to extract some information from our optical and IR data. The observed $V-R$ color for XTEJ1946+274 is $\sim 1.6$, while the intrinsic one should be $\sim-0.14 \rightarrow-0.11$ (assuming a main sequence or sub-giant star with spectral class in the B0-B1 III-V range; Wegner 1994), so the reddening should amount to $\sim 1.7 \rightarrow 1.8$. Assuming a standard reddening law (Fitzpatrick 1999) this converts to $A_{R} \sim 5.0 \rightarrow 5.1, A_{V} \sim 6.7 \rightarrow 6.8$ and $E_{B-V} \sim 2.0 \rightarrow 2.2$ (regardless of the distribution of the medium responsible for the reddening along the line of sight and near the source, i.e. assuming that the reddening due to circumstellar disk is negligible).

However from the $\mathrm{X}$-ray spectral data (Campana et al. 1998) a $N_{H}$ of $\sim 1.6 \times 10^{22} \mathrm{~cm}^{-2}$ was inferred, corresponding to an $E_{B-V} \sim 2.9$ (Predehl \& Schmitt 1995), which is slightly more than what obtained from optical data. Considering the total Galaxy $N_{H}$ column in the direction of XTE J1946+274 (Dickey \& Lockman 1990), $<1 \times 10^{22} \mathrm{~cm}^{-2}$, a value of $E_{B-V} \sim$ $1.9 \rightarrow 2.1$ was inferred, which comfortably overlaps the value inferred from the optical data. This result suggests that at least part of the inferred X-ray $N_{H}$ is local to the system and obscures the neutron star during outbursts. A good agreement with the $B$ to $I$ measurements is obtained for a B0-1IV-V star at a distance of about 8-10 kpc and with $E_{B-V} \sim 2$ (see Table 2). We can reasonably discard the possibility of a luminosity class III which would imply an $E_{B-V}>2.3$ and a large IR-deficiency. For a reference B0-1IV-V star $\left(M_{V} \sim-3.5\right)$ at a distance of 8-10 kpc and based on the IR photometry we infer an excess of $\sim 0.4,0.5$ and 1.4 mag in $J, H$ and $K$ filters respectively, suggesting the presence of a circumstellar envelope. Finally we note that in the direction of XTEJ1946+274 there are two spiral arms of our Galaxy located at $\sim 4 \mathrm{kpc}$ and $\sim 9 \mathrm{kpc}$ away from the Earth (Perseus and Cygnus arms; Turner 1980; Taylor \& Cordes 1993; Vallée 2002), so XTE J1946+274 could belong to the second one. Moreover this distance estimation is very similar to that found for the nearby source KS 1947+300 (Negueruela et al. 2002), which could lie on the same arm.

For a distance of $8-10 \mathrm{kpc}$ and a $1-10 \mathrm{keV}$ flux of $\sim 1 \times$ $10^{-9} \mathrm{erg} \mathrm{s}^{-1} \mathrm{~cm}^{-2}$ at the peak of the 1998 outburst (Takeshima \& Chakrabarty 1998) we obtain an X-ray luminosity of $L_{X}(1-$ $10 \mathrm{keV}) \simeq 8-20 \times 10^{36} \mathrm{erg} \mathrm{s}^{-1}$. Such a luminosity is a typical value shown by $\mathrm{X}$-ray pulsars in binary systems during Type I outbursts (Stella et al. 1986; Negueruela 1998) occurring close to the time of periastron passage and with a periodic recurrence at the orbital period of the system.

Based on both X-ray and optical/IR observations we identify the likely optical/IR counterpart of XTE J1946+274. Moreover, photometric and spectroscopic optical/IR data allows us to conclude that the proposed optical counterpart is most likely a B0-1 V-IVe star at a distance of about $\sim 8-10 \mathrm{kpc}$. A more accurate distance and spectral classification would require more detailed optical/IR spectroscopic observations.

Acknowledgements. This work is partially based on observations carried out at Loiano observing station (Bologna Observatory). The WHT is operated on the island of La Palma by the Isaac Newton Group in the Spanish Observatorio del Roque de los Muchachos of the Instituto de Astrofísica de Canarias. We thank the service programme for performing the observations. The United Kingdom Infrared Telescope is operated by the Joint Astronomy Centre on behalf of the U.K. Particle Physics and Astronomy Research Council. JSC acknowledges funding support from PPARC. Moreover authors would like to thank the referee, Dr. L. M. Kuiper, for useful advices.

\section{References}

Andrillat, Y., Jaschek, M., \& Jaschek, C. 1988, A\&AS, 72, 129

Bregoli, G., Federici, L., Merighi, R., et al. 1987, in ESO-OHP Workshop on the Optimization of the Use of CCD Detectors in Astronomy, Saint-Michel-1'Observatoire, France, June 17-19, 1986, Proc. (A88-13301 03-89), Garching, ESO, Germany, 177

Campana, S., Israel, G. L., Stella, L., \& Santangelo, A. 1998, IAU Circ., \#7039

Campana, S., Israel, G. L., \& Stella, L. 1999, A\&A, 352, L91 
Chakrabarty, D., Wang, Z., Juett, A. M., et al. 2002, ApJ, in press [astro-ph/0203124]

Clark, J. S., Steele, I. A., Fender, R. P., \& Coe, M. J. 1999, A\&A, 348, 888

Clark, J. S., \& Steele, I. A. 2000, A\&AS, 141, 65

Coe, M. J. 2000, Proc. of the 175th IAU Colloq., ed. M. A. Smith, H. F. Henrichs, \& J. Fabregat, ASP Conf. Ser., 214, 656

Covino, S., Israel, G. L., Polcaro, V. F., et al. 2000, Proc. of the 175th IAU Colloq., ed. M. A. Smith, H. F. Henrichs, \& J. Fabregat, ASP Conf. Ser., 214, 681

Covino, S., Negueruela, I., Campana, S., et al. 2001, A\&A, 374, 1009

Dickey, J. M., \& Lockman, F. J. 1990, ARA\&A, 28, 215

Fiore, F., Giommi, P., Vignali, C., et al. 2001, MNRAS, 327, 771

Fitzpatrick, E. L. 1999, PASP, 111, 63

Ghavamian, P., \& Garcia, M. 1998, IAU Circ., \#7022

Hanuschik, R. W. 1996, A\&A, 308, 170

Heindl, W. A., Coburn, W., Gruber, D. E., et al. 2001, ApJ, 563, L35

Israel, G. L., Polcaro, V. F., \& Covino, S. 1998, IAU Circ., \#7021

Israel, G. L., Covino, S., Campana, S., et al. 2000a, MNRAS, 314, 87

Israel, G. L., Polcaro, V. F., Covino, S., et al. 2000b, Proc. of the 175th IAU Colloq., ed. M. A. Smith, H. F. Henrichs, \& J. Fabregat, ASP Conf. Ser., 214, 739

Israel, G. L., Negueruela, I., Campana, S., et al. 2001, A\&A, 371, 1018 Merighi, R., Mignoli, M., Ciattaglia, C., et al. 1994, Bologna Technical Reports 09-1994-05

Negueruela, I. 1998, A\&A, 338, 505

Negueruela, I., Israel, G. L., Marco, A., Norton, A. J., \& Speziali, R. 2002, A\&A, submitted
Negueruela, I., \& Coe, M. J. 2002, A\&A, 385, 517

Nelson, R. W., Salpeter, E. E., \& Wasserman, I. 1993, ApJ, 418, 874

Nelson, R. W., Wang, W., Salpeter, E. E., \& Wasserman, I. 1995, ApJ, 438, L99

Okazaki, A. T. 2001, PASJ, 53, 119

Okazaki, A. T., \& Negueruela, I. 2001, A\&A, 377, 161

Paul, B., Agrawal, P. C., Mukerjee, K., et al. 2001, A\&A, 370, 529

Porter, J. M. 1999, A\&A, 348, 512

Predehl, P., \& Schmitt, J. H. M. M. 1995, A\&A, 293, 889

Puxley, P. J., Beard, S. M., \& Ramsay, S. K. 1992, in Data Analysis Workshop-4th ESO/ST-ECF Garching, 117

Smith, D. A., \& Takeshima, T. 1998, IAU Circ., \#7014

Stella, L., White, N. E., \& Rosner, R. 1986, ApJ, 208, 669

Stetson, P. B. 1987, PASP, 99, 191

Takeshima, T., \& Chakrabarty, D. 1998, IAU Circ., \#7016

Taylor, J. H., \& Cordes, J. M. 1993, ApJ, 411, 674

Turner, D. G. 1980, ApJ, 235, 146

Vallée, J. P. 2002, ApJ, 566, 261

Warwick, R. S., Marshall, N., Fraser, G. W., et al. 1981, MNRAS, 197, 865

Wegner, W. 1994, MNRAS, 270, 229

White, N. E., Nagase, F., \& Parmar, A. N. 1995, in X-ray Binaries, ed. W. H. G. Lewin, J. van Paradijs, \& E. P. J. van den Heuvel (Cambridge University Press), 1

Wilson, C. A., Finger, M. H., Wilson, R. B., \& Scott, D. M. 1998, IAU Circ., \#7014 